\title{
Complicated carbapenem-resistant infections: a treatment pathway analysis in Italian sites
}

\author{
Emanuele Durante-Mangoni ${ }^{1}$, Lorenzo Bertolino' ${ }^{1}$, Claudio Mastroianni ${ }^{2}$, Pierluigi Viale ${ }^{3}$, \\ Matteo Bassetti ${ }^{4}$, Rita Citton ${ }^{5}$, David Gómez-Ulloa ${ }^{6}$, Montse Roset ${ }^{6}$, Eilish McCann ${ }^{7}$ \\ ${ }^{1}$ Department of Precision Medicine and Unit of Infectious and Transplant Medicine, Università della Campania \\ 'L. Vanvitelli', Ospedale Monaldi, AORN Ospedali dei Colli, Naples, Italy; \\ ${ }^{2}$ Department of Infectious Diseases, Azienda Policlinico Umberto I, Rome, Italy; \\ 3Infectious Diseases Unit, Department of Medical and Surgical Sciences, AOU Policlinico S. Orsola-Malpighi, \\ Bologna, Italy; \\ 4Infectious Diseases Unit, Ospedale Policlinico San Martino IST- IRCCS, Genova, Italy \\ ${ }^{5}$ Medical Affairs, MSD, Rome, Italy; \\ ${ }^{6}$ Real World Solutions, IQVIA, Barcelona, Spain; \\ 7Outcomes Research, Merck \& CO., Inc., Kenilworth NJ, USA
}

Article received 24 March, 2021; accepted 2 July, 2021

\section{SUMMARY}

Introduction: Efforts to curb a growing prevalence of carbapenem resistance are prominent worldwide and especially in countries where high levels of carbapenem resistance are reported, such as Italy. Complicated infections, including complicated urinary tract infections (cUTI), complicated intra-abdominal infections (cIAI), and hospital-acquired/ventilator-associated bacterial pneumonia (HABP/VABP), are often caused by carbapenem-resistant Gram-negative (CRGN) bacteria and as such, these infection sites and their causative bacteria are important areas of focus for healthcare practitioners seeking to follow good antimicrobial stewardship practices. The aim of this study was to assess the clinical management and associated clinical and economic outcomes of patients with cUTI, cIAI, and $\mathrm{HABP} / \mathrm{VABP}$ resulting from CRGN bacteria in Italy.

Methods: We first conducted a hospital survey focusing on Gram-negative infections and their antibacterial susceptibility profile in four participating Italian hospitals. The second part of the study involved a non-interventional, retrospective single cohort chart review of 100 patients with cUTI, cIAI, or HABP/VABP caused by CRGN bacteria, in which patient characteristics, index hospitalization characteristics, infection characteristics, patient outcomes, treatment pathways, and healthcare resource use were assessed.

Results: The hospital survey demonstrated carbapenem resistance in approximately $17 \%$ of complicated infections, mostly associated with Acinetobacter baumannii. The non-interventional, retrospective cohort component showed that complicated CRGN infections were hospital- or healthcare-acquired in $99.0 \%$ of cases and were most often caused by Klebsiella pneumoniae $(66.0 \%)$. Despite the carbapenem-resistant nature of the included infections, carbapenems were used in $19.0 \%$ of patients as empirical therapy, in $43.0 \%$ as late empirical (i.e. immediately before receipt of susceptibility test results), and in $64.0 \%$ as targeted therapy (post-susceptibility test result receipt). Colistin was used in $61.0 \%$ of patients after susceptibility results were available. High clinical and economic burden was evident, with the average length of hospital stay being greater than 50 days, clinical cure achievement in only $43.0 \%$ of patients, and an overall mortality rate of $65.0 \%$ by the end of the follow-up period.

Conclusion: Our results reflect the considerable burden associated with complicated CRGN infections in Italy and the limitations in current treatment strategies. Our study pinpoints potential areas for improvement. For example, regular and detailed local surveillance and state of the art microbial diagnostic capabilities might aid and hasten clinical decision-making and facilitate improved antimicrobial stewardship when treating complex CRGN infections. New therapeutic options which more appropriately address CRGN infections may assist in improving outcomes which are important to both patients and healthcare providers.

Keywords: Antimicrobial stewardship, carbapenem resistance, complicated infections, Gram-negative infections, healthcare-associated infections. 


\section{INTRODUCTION}

A ntibacterial resistance is recognized as a worldwide issue, associated with increased length of hospital stay, mortality, and overall healthcare costs [1]. Efforts to treat antibacterial-resistant infections properly and to prevent further development of antibacterial resistance have been the focus of many governments and organizations in recent years [2]. This is of particular importance when considering resistance to hitherto frequently used classes of antibacterial agents such as carbapenems. Several carbapenem-resistant pathogens are categorized as "critical" in the World Health Organization's global priority list because of their alarming spread, especially in southern European countries $[3,4]$ [2]. Carbapenem resistance has been reported to be high in Italy: in 2017 19.9\% of P. aeruginosa, $29.7 \%$ of K. pneumoniae and $78.7 \%$ of A. baumannii were resistant [3]. Furthermore, KPC-producing K. pneumoniae are considered to be endemic in the country and account for nearly all $(95.1 \%)$ of the carbapenemase-producing Enterobacterales bloodstream infections $[5,6]$. There has also been a recent emergence of bacterial strains with resistance to colistin, an antibacterial agent that can be used as a last-resort option for carbapenem-resistant (CR) infections but with considerable limitations, such as difficulty in achieving optimal dosing, high levels of renal toxicity, and problems in determining bacterial susceptibility $[7,8]$. There is a difficult balance to strike when trying to treat patients with complicated infections both quickly and appropriately, and limitations in available treatments due to bacterial resistance may further complicate clinical decision-making [9]. A better understanding of current diagnostic and treatment practices is needed to help identify potential areas for improvement in terms of improved antimicrobial stewardship and clinical and economic outcomes for CR Gram-negative (CRGN) infections in Italy. The objective of this study was to characterize the clinical management of patients diagnosed with complicated urinary tract infections (cUTI), complicated intra-abdominal infections (cIAI), and hospital-acquired/ventilator-as-

\section{Corresponding author}

Emanuele Durante-Mangoni

E-mail: emanuele.durante@unicampania.it sociated bacterial pneumonia (HABP/VABP) attributable to CRGN bacteria in Italy.

\section{PATIENTS AND METHODS}

Four hospitals participated in the study; the sites were all large (greater than 600 beds) teaching facilities. Carbapenem resistance was defined as per the European Committee on Antimicrobial Susceptibility Testing (EUCAST) susceptibility cutoff values in use at the time of culture collection [10]. Resistant or intermediate isolates were defined as $\mathrm{CR}$ and permitted inclusion into the study.

\section{Preliminary survey}

Hospital microbiological surveillance data from 2015-2017 were evaluated through a questionnaire. The number of cUTI, cIAI, and HABP/VABP infections per year, associated causative pathogens, and antibacterial resistance profiles were collected, by infection site.

\section{Retrospective observational study}

A non-interventional, retrospective, single cohort chart review was conducted to assess patients with cUTI, cIAI, or HABP/VABP attributable to CRGN bacteria in the same hospitals. Patients were enrolled by treating physicians in a retrospective manner from July 2017 backwards through a 30-month patient identification window period until a target number of patients $(\mathrm{N}=100)$ was reached.

Patients were eligible for inclusion if they were at least 18 years old, admitted to the hospital during the patient identification window period, had a confirmed diagnosis of cUTI, cIAI, or HABP/ VABP caused by a CRGN bacteria (based on a culture collected during their hospital stay), and had all medical data available from the date of culture collection to the end of follow-up (until date of death or up to 6 months after hospital discharge). A clinical diagnosis by the physician and a confirmatory culture for CRGN bacteria were mandatory to consider a potential case as an infection. Patients participating in a clinical trial during the observation period were excluded. The study was approved by the Ethics Committees of all participating sites. Italian regulations related to patient consent were followed, and signed patient consents were collected as appropriate prior to enrolment. 


\section{Study variables}

Study variables included patient characteristics, index hospitalization characteristics, infection characteristics, details of treatment pathways, patient outcomes, and healthcare resource use during the index hospitalization. Patient characteristics collected were age, sex, body mass index (BMI), smoking status, alcohol consumption, comorbidities, hypersensitivity to antibacterial agents, reason for admission, ward of admission, and previous medical history, specifically prior nursing home admission, prior hospital stays, and invasive procedures and antibacterial agents received during the year prior to index hospitalization. Index hospitalization characteristics included length of stay, intensive care unit (ICU) need, ICU length of stay, and APACHE II (Acute Physiology And Chronic Health Evaluation II) score at ICU admission for those who were admitted to the ICU. Infection characteristics collected were infection site, presence of secondary bacteraemia, place of infection acquisition, pathogen isolated per patient, pathogen verification method, antibacterial susceptibility verification method and results, carbapenemase verification method and results, and isolate source.
Treatment pathway details included the antibacterial agents prescribed at culture collection, prior to culture susceptibility test results, and after culture susceptibility results. Healthcare resource use was described by the number of diagnostic tests and invasive procedures recorded during index hospitalization. Patient outcome variables collected included clinical cure and time to clinical cure (i.e. from culture collection to complete resolution of all signs and symptoms of infection). The EU-CARE study has provided novel evidence in the presentation of the flow treatments received by patients at 3 different cross-sectional timepoints: at culture collection or next day (early empiric therapy), prior to culture susceptibility results (late empiric therapy), and after culture susceptibility results (first targeted therapy). Early empiric therapy corresponds to the first antibiotic treatment(s) that the patient received at date of culture collection or the day after but before susceptibility was known. Prior to culture susceptibility test results (late empiric therapy) includes those treatment(s) initiated between day 2 after culture collection and date of availability of antibiogram susceptibility results. And finally, first

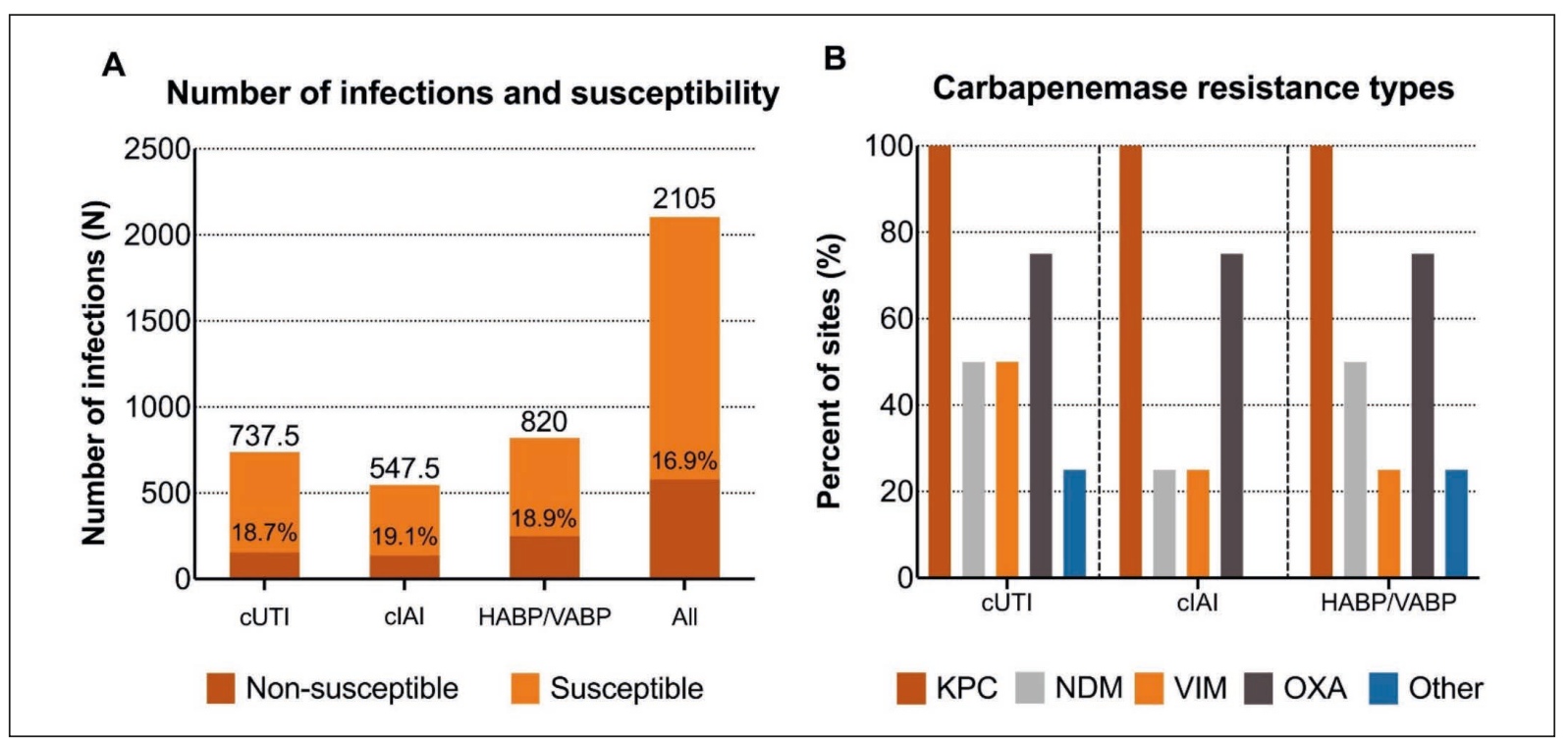

Figure 1 - Hospital reported local Gram-negative infection characteristics.

Note: Data based on initial assessment performed by the sites $(n=5)$ regarding the number and type of complicated Gram-negative infections over the last 3 years.

Figure 1A shows mean number of infections, colors represent ratio of antibiotic susceptibility/non-susceptibility.

Figure $1 \mathrm{~B}$ shows percentage of hospital sites that reported different types of resistance with different infection types.

cIAI: Complicated intra-abdominal infection; cUTI: Complicated urinary tract infection; GN: Gram-negative; HABP/VABP: Hospital-acquired bacterial pneumonia/ventilator-associated bacterial pneumonia; KPC: Klebsiella pneumoniae carbapenemase; NDM: New Delhi metallo-beta-lactamase; OXA: Oxacillinase type; VIM: Verona Integron-Mediated metallo-beta-lactamase. 
targeted therapy corresponds to treatment(s) prescribed after culture susceptibility results. Hereafter, first targeted therapy has been identified based on the following criteria:

a) treatment start date is after the date of antibiogram availability and no later than two days after the date of antibiogram availability;

b) treatment start date is prior to the date of antibiogram availability and treatment end date is at minimum two days after the date of antibiogram availability;

c) if no treatments are reported as per criteria defined above, the first treatment received between 3 and 7 days (inclusive) after the date of antibiogram availability is considered.

If other antibiotic treatments are initiated maximum 1 day after first antibiotic treatment start date, these are also considered as part of a combination therapy regimen. Additional variables collected were presence and duration of severe sepsis/septic shock, mortality during index hospitalization and during the follow-up period, time to discharge, and the time to, and number of, readmissions for any diagnosis. Recurrence was defined as the development of new symptoms and signs of infection in association with a culture positive for CRGN following initial response to antibiotic therapy.

\section{Statistical analysis}

Descriptive statistics were analyzed with SAS Enterprise Guide version 7.13. Continuous variables are described as mean values and their standard deviations (SD), categorical variables are described by number and percentage. Furthermore, a subset analysis was performed on patients receiving carbapenems at any timepoint during index hospitalization $(n=67)$ in order to assess their treatment pathways. Missing data were not included or imputed in the calculations, except for missing dates, where missing day values were substituted with mid-month dates in order to calculate length of time in days.

\section{RESULTS}

\section{Preliminary survey}

Hospitals reported an average of $737.5(\mathrm{SD}=430.8)$ cUTI, 547.5 (SD=452.29) cIAI, and $820.0(\mathrm{SD}=385.1)$ HABP/VABP cases (Figure 1A, Supplementary Table 1) in the three years prior to the survey. Al- together $16.9 \%$ of isolates were $C R$, with a similar frequency of $\mathrm{CR}$ levels across all three infection sites. KPC was the most common carbapenemase type observed in those isolates which were tested for carbapenemase production, with all participating hospitals reporting KPC in all three infection sites (Figure 1B, Supplementary Table 1). Oxacillinase type carbapenemase (OXA) was

Supplementary Table 1 (data for Figure 1) - Hospital reported local Gram-negative infection characteristics.

\begin{tabular}{|c|c|c|}
\hline Infection type & Characteristic & No. $(\%)$ \\
\hline \multirow[t]{3}{*}{ cUTI } & $\begin{array}{l}\text { Total Gram-negative } \\
\text { infections - mean (SD) }\end{array}$ & 737.5 (430.8) \\
\hline & $\begin{array}{l}\text { Carbapenem-resistant } \\
\text { (\% of total isolates) }\end{array}$ & $21(18.7 \%)$ \\
\hline & $\begin{array}{l}\text { Resistance type* }^{*} \\
\text { KPC } \\
\text { NDM } \\
\text { VIM } \\
\text { OXA } \\
\text { Other }\end{array}$ & $\begin{array}{l}4(100 \%) \\
2(50.0 \%) \\
2(50.0 \%) \\
3(75.0 \%) \\
1(25.0 \%)\end{array}$ \\
\hline \multirow[t]{3}{*}{ cIAI } & $\begin{array}{l}\text { Total Gram-negative } \\
\text { infections }\end{array}$ & $547.5(452.2)$ \\
\hline & $\begin{array}{l}\text { Carbapenem resistant } \\
\text { (\% of total isolates) }\end{array}$ & $25(19.1 \%)$ \\
\hline & $\begin{array}{l}\text { Resistance type }^{*} \\
\text { KPC } \\
\text { NDM } \\
\text { VIM } \\
\text { OXA } \\
\text { Other }\end{array}$ & $\begin{array}{c}4(100 \%) \\
1(25 \%) \\
1(25 \%) \\
3(75 \%) \\
0(0 \%)\end{array}$ \\
\hline \multirow[t]{3}{*}{ HABP/VABP } & $\begin{array}{l}\text { Total Gram-negative } \\
\text { infections }\end{array}$ & $820.0(385.1)$ \\
\hline & $\begin{array}{l}\text { Carbapenem resistant } \\
\text { (\% of total isolates) }\end{array}$ & $30.5(18.9 \%)$ \\
\hline & $\begin{array}{l}\text { Resistance type }^{*} \\
\text { KPC } \\
\text { NDM } \\
\text { VIM } \\
\text { OXA } \\
\text { Other }\end{array}$ & $\begin{array}{l}4(100 \%) \\
2(50 \%) \\
1(25 \%) \\
3(75 \%) \\
1(25 \%)\end{array}$ \\
\hline $\begin{array}{l}\text { cUTI + cIAI + } \\
\mathrm{HABP} / \mathrm{VABP}\end{array}$ & $\begin{array}{l}\text { Carbapenem resistant } \\
\text { (\% of total isolates) }\end{array}$ & $27.5(16.9 \%)$ \\
\hline
\end{tabular}

Note: Data based on initial assessment performed by the sites $(n=4)$ regarding the number and type of complicated Gram-negative infections over the last 3 years. Data are presented as mean (SD) or $n(\%)$.

*Multi-response variable. Data shows number (\%) of sites that reported type of resistance.

cIAI: Complicated intra-abdominal infection; cUTI: Complicated urinary tract infection; GN: Gram-negative; HABP/VABP: Hospitalacquired bacterial pneumonia/ventilator-associated bacterial pneumonia; KPC: Klebsiella pneumoniae carbapenemase; NDM: New Delhi metallo-beta-lactamase; OXA: Oxacillinase type; VIM: Verona IntegronMediated metallo-beta-lactamase. 
also common with three of the four hospital sites reporting OXA across all infection sites; New Delhi metallo-beta-lactamase (NDM) and Verona Integron-Mediated metallo-beta-lactamase (VIM) were less frequently identified.

Carbapenem resistance was highest for A. baumannii isolates (40\%-70\%) and $K$. pneumoniae isolates (20\%-43\%) (Figure 2, Supplementary Table 2). Escherichia coli, Pseudomonas aeruginosa, and other Enterobacterales isolates were less likely to be resistant to carbapenems (note: P. aeruginosa has endogenous resistance towards ertapenem). However, resistance was common towards other antibacterial groups: $38.3-72.5 \%$ against penicillins and 29.3$64.3 \%$ against fluoroquinolones, amongst isolates.

\section{Retrospective observational study}

\section{Patient characteristics}

Patients were mostly male, with an average age of $64.1(\mathrm{SD}=16.6)$ years (Table 1$)$. Almost a third were overweight. Smoking and heavy drinking were uncommon. At least one comorbidity was noted for $94.0 \%$ of patients. Chronic lung disease, hypertension, and diabetes were each present in about a quarter of patients, and renal disease was also common. Nursing home stay was not reported frequently, but more than half of patients had a documented hospital stay in the year prior to index hospitalization. Altogether $38.0 \%$ of patients had received a prior course of antibacterial agents, most commonly penicillins $(n=24)$. Prior carbapenem use was recorded for 11 patients. At admission, the most common diagnosis was infection or a respiratory disease; over half of patients were admitted to medical wards and a third to surgical wards.

Index hospitalization characteristics

Mean length of stay was 52.9 ( $\mathrm{SD}=0.5)$ days, with HABP/VABP patients having the longest hospitalization time (Figure 3, Supplementary Table 3).

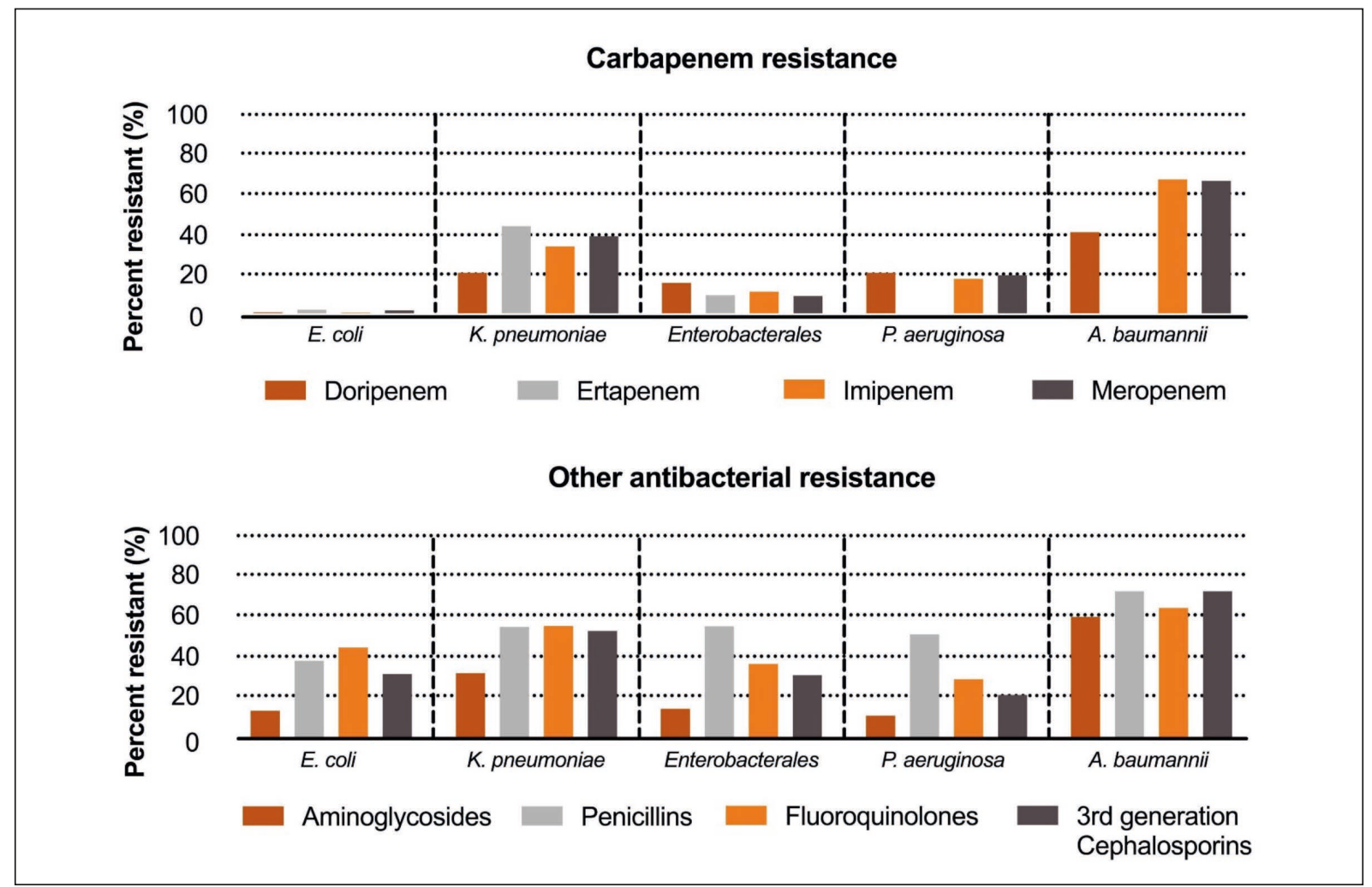

Figure 2 - Antibiotic resistance for different pathogens - Percentage of isolates that were resistant to each antibiotic class in pathogens observed in 2017.

Note: percent resistance refers to the percent non-susceptible (intermediate or resistant). Ertapenem resistance is not shown for Pseudomonas and Acinetobacter isolates, since these bacteria are intrinsically resistant to this antibiotic. 
ICU need was high for all infection sites (38.2\%$62.5 \%$ ) but was highest for patients with HABP / VABP, though patients with cUTI stayed in the ICU for the longest period (28.0 [SD=23.0] days). APACHE II scores at ICU admission, for those

Supplementary Table 2 (data for Figure 2) - Antibacterial resistance for different pathogens.

\begin{tabular}{|c|c|c|}
\hline Isolate & Antibacterial agent & $\begin{array}{c}\text { Mean (SD) } \\
\text { percentage of } \\
\text { non-susceptible } \\
\text { isolates in five } \\
\text { hospitals }\end{array}$ \\
\hline E. coli & $\begin{array}{l}\text { Aminoglycosides } \\
\text { Penicillins } \\
\text { Doripenem } \\
\text { Ertapenem } \\
\text { Imipenem } \\
\text { Meropenem } \\
\text { Fluoroquinolones } \\
\text { 3rd gen cephalosporins }\end{array}$ & $\begin{array}{c}13.8(8.9) \\
38.3(22.2) \\
0.5(0.7) \\
1.8(2.9) \\
0.3(0.6) \\
1.5(2.4) \\
44.8(12.5) \\
31.8(12.2)\end{array}$ \\
\hline K. pneumoniae & $\begin{array}{l}\text { Aminoglycosides } \\
\text { Penicillins } \\
\text { Doripenem } \\
\text { Ertapenem } \\
\text { Imipenem } \\
\text { Meropenem } \\
\text { Fluoroquinolones } \\
\text { 3rd gen cephalosporins }\end{array}$ & $\begin{array}{l}32.3(22.2) \\
55.0(23.9) \\
20.0(21.2) \\
43.0(29.4) \\
33.0(27.1) \\
38.0(24.6) \\
55.5(18.4) \\
53.3(23.4) \\
\end{array}$ \\
\hline $\begin{array}{l}\text { Other } \\
\text { Enterobacterales } \\
\text { spp. }\end{array}$ & $\begin{array}{l}\text { Aminoglycosides } \\
\text { Penicillins } \\
\text { Doripenem } \\
\text { Ertapenem } \\
\text { Imipenem } \\
\text { Meropenem } \\
\text { Fluoroquinolones } \\
\text { 3rd gen cephalosporins }\end{array}$ & $\begin{array}{c}14.8(11.5) \\
55.3(26.8) \\
15.0(14.1) \\
9.0(10.7) \\
10.7(12.5) \\
8.5(11.1) \\
36.8(19.2) \\
31.3(11.1)\end{array}$ \\
\hline P. aeruginosa & $\begin{array}{l}\text { Aminoglycosides } \\
\text { Penicillins } \\
\text { Doripenem } \\
\text { Ertapenem } \\
\text { Imipenem } \\
\text { Meropenem } \\
\text { Fluoroquinolones } \\
\text { 3rd gen cephalosporins }\end{array}$ & $\begin{array}{c}11.3(12.7) \\
51.3(32.8) \\
20.0(7.1) \\
100.0(0.0) \\
17.0(10.6) \\
18.8(9.7) \\
29.3(4.6) \\
21.5(6.2)\end{array}$ \\
\hline A. baumannii & $\begin{array}{l}\text { Aminoglycosides } \\
\text { Penicillins } \\
\text { Doripenem } \\
\text { Ertapenem } \\
\text { Imipenem } \\
\text { Meropenem } \\
\text { Fluoroquinolones } \\
\text { 3rd gen cephalosporins }\end{array}$ & $\begin{array}{l}60.0(39.5) \\
72.5(48.6) \\
40.0(56.6) \\
70.0(47.6) \\
65.8(44.5) \\
65.3(44.2) \\
64.3(43.6) \\
72.5(48.6)\end{array}$ \\
\hline
\end{tabular}

Note: Percentage of isolates that were non-susceptible (intermediate or resistant) to each antibacterial agent class in pathogens observed in 2017.
Table 1 - Patient characteristics recorded at hospital admission.

\begin{tabular}{|c|c|}
\hline Patient characteristics & No. $(\%)$ \\
\hline \multicolumn{2}{|l|}{ At baseline } \\
\hline Age, years - mean (SD) & $64.1(16.6)$ \\
\hline $\begin{array}{l}\text { Sex } \\
\text { Female } \\
\text { Male }\end{array}$ & $\begin{array}{l}36(36.0 \%) \\
64(64.0 \%)\end{array}$ \\
\hline $\begin{array}{l}\text { BMI categories } \\
<18.5 \mathrm{~kg} / \mathrm{m}^{2} \\
18.5-25 \mathrm{~kg} / \mathrm{m}^{2} \\
>25 \mathrm{~kg} / \mathrm{m}^{2}\end{array}$ & $\begin{array}{l}10(10.0 \%) \\
32(32.0 \%) \\
29(29.0 \%)\end{array}$ \\
\hline $\begin{array}{l}\text { Smoking status } \\
\text { Never smoked } \\
\text { Previous smoking } \\
\text { Current smoker }\end{array}$ & $\begin{array}{l}23(23.0 \%) \\
18(18.0 \%) \\
10(10.0 \%) \\
\end{array}$ \\
\hline $\begin{array}{l}\text { Alcohol consumption } \\
\text { Never } \\
\text { Occasional } \\
\text { Heavy }\end{array}$ & $\begin{array}{c}20(20.0 \%) \\
12(12.0 \%) \\
1(1.0 \%)\end{array}$ \\
\hline $\begin{array}{l}\text { Comorbidities* }^{*} \\
\text { Chronic lung disease } \\
\text { Diabetes } \\
\text { Hypertension } \\
\text { Renal disease } \\
\text { Solid tumor } \\
\text { None } \\
\text { Hypersensitivity to antibiotics }\end{array}$ & $\begin{array}{c}25(25.0 \%) \\
25(25.0 \%) \\
24(24.0 \%) \\
20(20.0 \%) \\
16(16.0 \%) \\
6(6.0 \%) \\
3(3.0 \%) \\
\end{array}$ \\
\hline $\begin{array}{l}\text { In the year prior to index hospitalization } \\
\text { Hospital admission } \\
\text { Nursing home stay } \\
\text { Nursing home resident at admission } \\
\text { Invasive procedures } \\
\text { Previous antibiotic administration* } \\
\text { Penicillin } \\
\text { Carbapenem } \\
\text { Cephalosporins } \\
\text { Other }\end{array}$ & $\begin{array}{c}57(57.0 \%) \\
5(5.0 \%) \\
4(4.0 \%) \\
45(45.0 \%) \\
38(38.0 \%) \\
24(63.2 \%) \\
11(29.0 \%) \\
8(21.1 \%) \\
13(34.2 \%) \\
\end{array}$ \\
\hline \multicolumn{2}{|l|}{ At admission } \\
\hline $\begin{array}{l}\text { Reason for admission } \\
\text { Infection } \\
\text { Respiratory } \\
\text { Cardiovascular } \\
\text { Gastrointestinal } \\
\text { Neurological } \\
\text { Metabolic/renal } \\
\text { Trauma } \\
\text { Other }\end{array}$ & $\begin{array}{l}29(29.0 \%) \\
24(24.0 \%) \\
12(12.0 \%) \\
11(11.0 \%) \\
6(6.0 \%) \\
5(5.0 \%) \\
2(2.0 \%) \\
11(11.0 \%)\end{array}$ \\
\hline $\begin{array}{l}\text { Ward of admission } \\
\text { Medical } \\
\text { Surgical } \\
\text { ICU }\end{array}$ & $\begin{array}{l}53(53.0 \%) \\
33(33.0 \%) \\
14(14.0 \%)\end{array}$ \\
\hline
\end{tabular}

${ }^{*}$ Multi-response variable. 
patients that required ICU, were also highest for cUTI (19.7 [SD=10.7]).

\section{Infection characteristics}

The most common infection site included in the retrospective cohort was HABP/VABP, followed by cUTI and cIAI (Table 2). Secondary bacteraemia occurred in $44.0 \%$ of patients. The site of infection acquisition was either the hospital or an- other healthcare setting in nearly all cases, with only $1.0 \%$ of cases being community-acquired. The most common causative pathogen was $K$. pneumoniae, being implicated in two thirds of included patients. A. baumannii and $P$. aeruginosa were less common, and E. coli was only isolated from two patients. Bacterial verification was mostly performed via culture techniques, but MALDI-TOF was also used frequently (45.0\% of cases). Antibac-
A

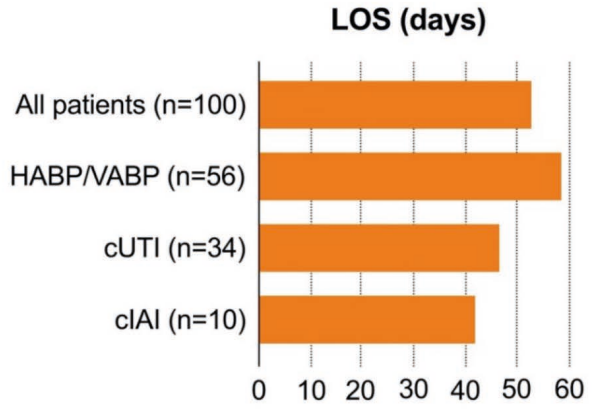

C

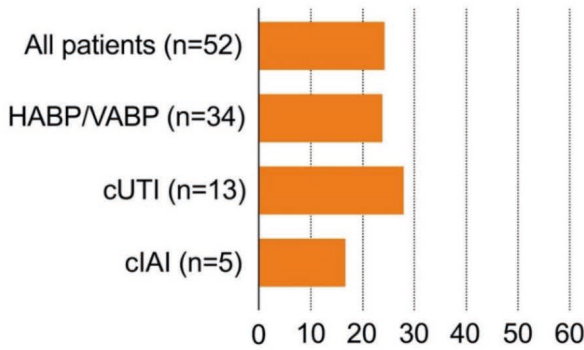

B

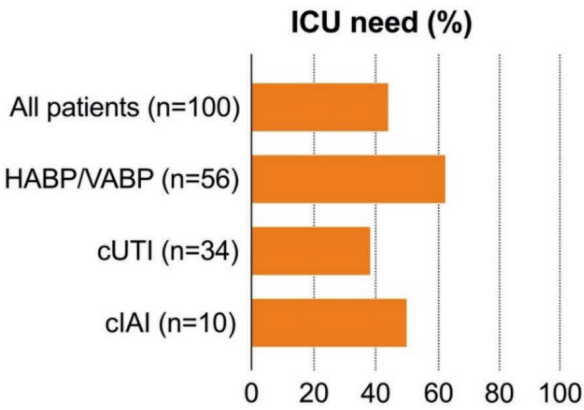

APACHE II score (points)

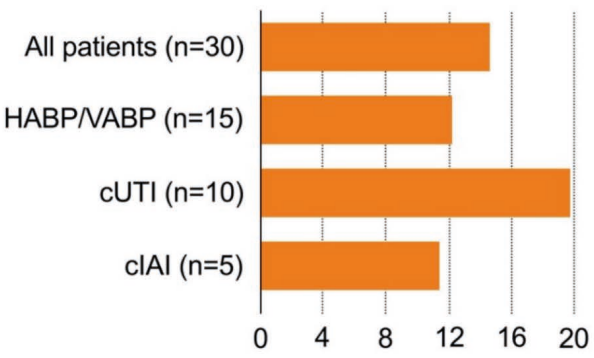

Figure 3 - Index hospitalization characteristics.

Note: A: Length of stay for different infection types (days). B: Intensive care unit need (percentage of all patients). C: Intensive care unit length of stay (days). D: APACHE II score. Worst score if more than one admission. Based on limited data (44\% of patient data missing). Data are presented as mean values. APACHE II: Acute Physiology And Chronic Health Evaluation II; cIAI: Complicated intra-abdominal infection; cUTI: Complicated urinary tract infection; HABP/VABP: Hospital-acquired bacterial pneumonia/ventilator-associated bacterial pneumonia; ICU: Intensive care unit; LOS: Length of stay; SD: Standard Deviation.

Supplementary Table 3 (data for Figure 3) - Index hospitalization characteristics.

\begin{tabular}{|l|c|c|c|c|}
\hline & $\begin{array}{c}\text { All patients } \\
(N=100)\end{array}$ & $\begin{array}{c}\text { HABP/VABP } \\
(N=56)\end{array}$ & $\begin{array}{c}\text { cUTI } \\
(\mathrm{N}=34)\end{array}$ & $\begin{array}{c}\text { cIAI } \\
(\mathrm{N}=10)\end{array}$ \\
\hline LOS (days) & $52.9(68.5)$ & $58.7(83.2)$ & $46.7(45.9)$ & $42.0(33.0)$ \\
\hline ICU need & $44(44.0 \%)$ & $35(62.5 \%)$ & $13(38.2 \%)$ & $5(50.0 \%)$ \\
\hline ICU LOS (days) & $24.3(20.4)$ & $23.9(20.2)$ & $28.0(23.0)$ & $16.8(16.3)$ \\
\hline APACHE II score & $14.6(8.6)$ & $12.2(6.9)$ & $19.7(10.7)$ & $11.4(3.1)$ \\
\hline
\end{tabular}

Note: Data are presented as mean (SD) or $\mathrm{n}(\%)$. ${ }^{*}$ Worse score if more than one admission. Based on limited data (43\% of patient data missing). APACHE II: Acute Physiology And Chronic Health Evaluation II; cIAI: Complicated intra-abdominal infection; cUTI: Complicated urinary tract infection; HABP/VABP: Hospital-acquired bacterial pneumonia/ventilator-associated bacterial pneumonia; ICU: Intensive care unit; LOS: Length of stay; SD: Standard Deviation. 
terial susceptibility was verified via an automated test or dilution methods in most cases, while E-test and disk diffusion methods were rarely used. The most common sources for isolates were urine and blood samples.

Figure 4 (see also Supplementary Table 4) demonstrates that most isolates were tested for susceptibility to meropenem or imipenem, while ertapenem susceptibility was less frequently tested, and doripenem susceptibility was only tested against a few isolates. All tested A. baumannii and most $P$. aeruginosa isolates were classified as resistant, while

Table 2 - Infection characteristics.

\begin{tabular}{|l|c|}
\hline \multicolumn{1}{|c|}{ Infection characteristics } & No. $(\%)$ \\
\hline Infection site & \\
HABP/VABP & $56(56.0 \%)$ \\
cUTI & $34(34.0 \%)$ \\
cIAI & $10(10.0 \%)$ \\
Secondary bacteraemia & $44(44.0 \%)$ \\
\hline Place of acquisition of infection* & \\
Hospital-acquired & $78(78.0 \%)$ \\
Healthcare-acquired & $21(21.0 \%)$ \\
Community-acquired & $1(1.0 \%)$ \\
\hline Pathogen isolated, per patient** & \\
K. pneumoniae & $66(66.0 \%)$ \\
A. baumannii & $23(23.0 \%)$ \\
P. aeruginosa & $12(12.0 \%)$ \\
E. coli & $2(2.0 \%)$ \\
\hline Pathogen verification ${ }^{* *}$ & \\
Culture & $80(80.0 \%)$ \\
MALDI-TOF & $45(45.0 \%)$ \\
\hline Antibiotic susceptibility verification $* *$ & \\
Automated antimicrobial susceptibility test & $42(42.0 \%)$ \\
Dilution & $26(26.0 \%)$ \\
E-test & $2(2.0 \%)$ \\
Disk diffusion & $2(2.0 \%)$ \\
\hline Sample type** & \\
Urine & $36(36.0 \%)$ \\
Blood & $36(36.0 \%)$ \\
Other & $27(27.0 \%)$ \\
BAL & $24(24.0 \%)$ \\
Sputum & $12(12.0 \%)$ \\
Peritoneal fluid & $4(4.0 \%)$ \\
Pleural fluid & $3(3.0 \%)$ \\
\hline
\end{tabular}

${ }^{*}$ Hospital-acquired: culture collected $=3$ days after admission; Healthcare-acquired: culture collected $<3$ days after admission with previous healthcare exposure; Community-acquired: culture collected $<3$ days after admission with no previous healthcare exposure.

** Multi-response variable.

BAL: Bronchoalveolar lavage; cIAI: Complicated intra-abdominal infection; cUTI: Complicated urinary tract infection; HABP/VABP: Hospital-acquired bacterial pneumonia/ventilator-associated bacterial pneumonia; MALDI-TOF: Matrix-assisted laser desorption ionizationtime of flight mass spectrometry; PCR: Polymerase chain reaction.

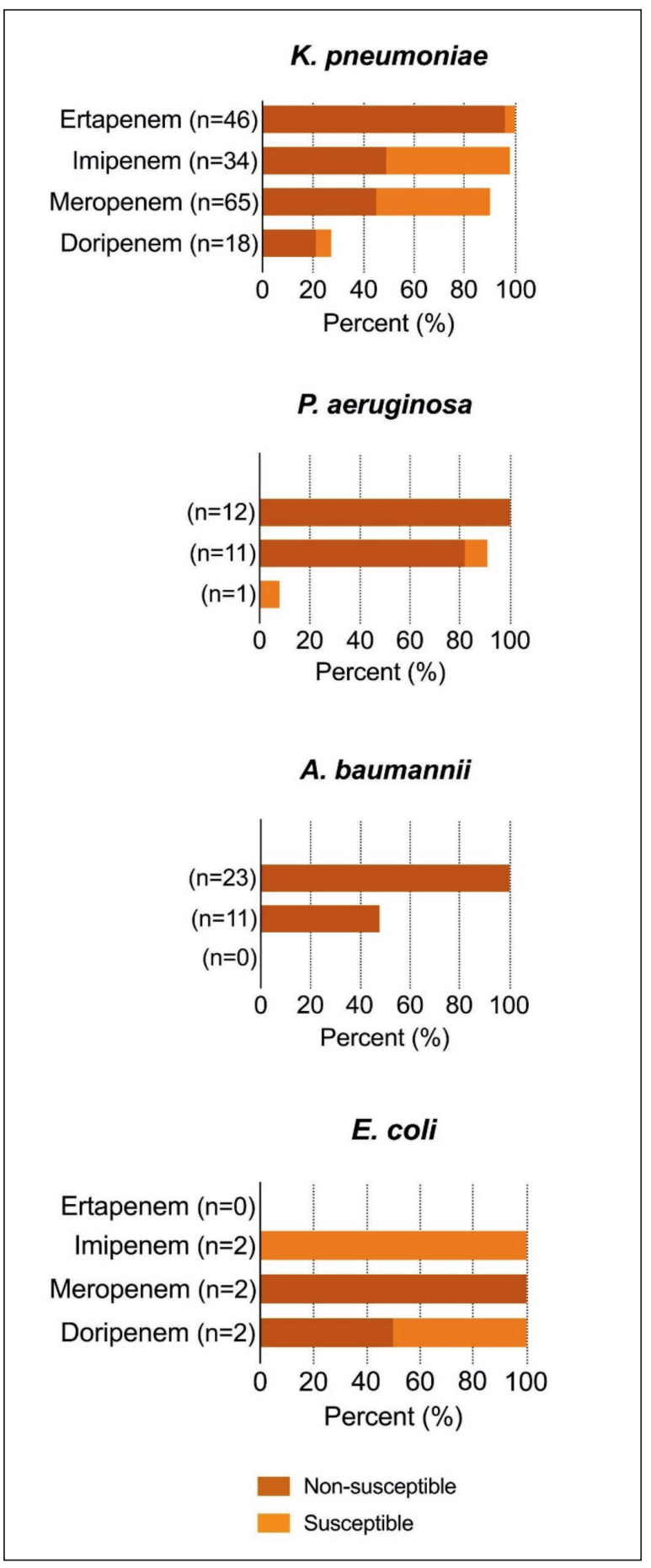

Figure 4 - Susceptibility test results.

Note: Susceptibility information for confirmatory cultures. Colors represent the proportion of isolates which are susceptible/non-susceptible; the height of columns indicates the percentage of isolates tested. Ertapenem resistance is not shown for Pseudomonas and Acinetobacter isolates, since these bacteria are intrinsically resistant to this antibiotic. 
Supplementary Table 4 (data for Figure 4) - Susceptibility test results.

\begin{tabular}{|l|c|c|c|c|c|c|c|c|}
\hline \multirow{2}{*}{} & \multicolumn{2}{|c|}{ Doripenem } & \multicolumn{2}{c|}{ Ertapenem } & \multicolumn{2}{c|}{ Imipenem } & \multicolumn{2}{c|}{ Meropenem } \\
\cline { 2 - 8 } & Tested & Resistant & Tested & Resistant & Tested & Resistant & Tested & Resistant \\
\hline K. pneumoniae & $18(27.3 \%)$ & $14(77.8 \%)$ & $46(69.7 \%)$ & $45(97.8 \%)$ & $34(51.5 \%)$ & $30(88.3 \%)$ & $65(98.5 \%)$ & $64(98.5 \%)$ \\
\hline P. aeruginosa & $1(8.3 \%)$ & $0(0 \%)$ & $8(66.7 \%)$ & $8(100 \%)$ & $12(100 \%)$ & $12(100 \%)$ & $11(91.7 \%)$ & $10(89.9 \%)$ \\
\hline A. baumannii & $0(0 \%)$ & N/A & $15(65.2 \%)$ & $15(100 \%)$ & $23(100 \%)$ & $23(100 \%)$ & $11(47.8 \%)$ & $11(100 \%)$ \\
\hline E. coli & $2(100 \%)$ & $1(50.0 \%)$ & $0(0 \%)$ & N/A & $2(100 \%)$ & $0(0 \%)$ & $2(100 \%)$ & $2(100 \%)$ \\
\hline
\end{tabular}

Note: Susceptibility information of confirmatory cultures related to carbapenem susceptibility. Data are presented as n (\%).

K. pneumoniae and E. coli isolates were more likely to be susceptible towards the carbapenems tested. Carbapenemase tests were performed on 34 of the 103 isolates, with all tested isolates confirmed to be producers of KPC. The majority of carbapenemase testing was performed via Xpert Carba- $R$ (73.5\%). Additionally, the boronic acid test (20.6\%) and Cepheid test $(2.9 \%)$ were also used.

\section{Treatment pathways}

At culture collection, the majority of patients $(n=52,52 \%)$ did not receive an antibacterial agent. Among those who did, monotherapy $(\mathrm{n}=19,19 \%)$ and combination (with two agents) ( $n=21,21 \%)$ was common, while administration of three or more antibacterial agents was rare $(n=7,7 \%)$. Prior to the availability of susceptibility results, the most common treatment type was combination therapy $(n=34,34 \%)$, no treatment $(n=27,27 \%)$, monotherapy $(n=20,20 \%)$, or three or more antibacterial agents $(n=19,19 \%)$. After antibiogram results were available, the majority of patients received combination therapy $(n=44,44 \%)$, followed by combination therapy with three or more antibiotics $(n=26,26 \%)$, while monotherapy $(n=21,21 \%)$ and no treatment $(n=8,8 \%)$ were less common. The most common antibacterial agents prescribed after culture collection were meropenem $(18.0 \%)$, colistin $(14.0 \%)$, and piperacillin (14.0\%). Immediately prior to the availability of susceptibility results, $41.0 \%$ of patients received meropenem, $25.0 \%$ colistin, and $18.0 \%$ piperacillin. After antibiogram results were available, meropenem and colistin were each administered to $61.0 \%$ of patients, and gentamicin and tigecycline to $14.0 \%$ and $13.0 \%$ of patients, respectively. Other antibiotics were prescribed less frequently at each timepoint (see Supplementary Table 5a).

Figure 5 shows treatment pathways for patients who received carbapenems at any timepoint $(\mathrm{n}=67)$ (in addition, see Supplementary Table $5 b$ ). As can be seen from the figure, immediately after culture collection more than half of patients $(n=35)$ did not receive any antibiotics and 37.3\% of patients $(n=25)$ received either one or two antibiotics, with the most common regimen being a carbapenem with an additional antibiotic $(n=8)$. By the second timepoint (immediately prior to receipt of susceptibility results), approximately half of patients $(n=33)$ received a carbapenem plus one or two other antibiotics. After receiving the susceptibility results, this proportion increased to $82.1 \%$ of patients $(n=55)$, while treatment combinations not involving any carbapenems became rare $(n=3)$. At that point, the most common agent in combination with carbapenems was colistin $(n=43)$. In addition, colistin (either alone or in combination) was also prescribed after receiving the susceptibility results to a further 18 patients not receiving carbapenems (data not shown in table or figure).

\section{Healthcare resource use}

Table 3 shows data relating to healthcare resource use during index hospitalization. Blood cell count and biochemistry tests were the most common tests performed, but blood cultures, radiographic investigations, and urine cultures were also performed in at least $40.0 \%$ of patients. Endoscopy was the most common invasive procedure, followed by vascular catheterization, and percutaneous drainage.

\section{Patient outcomes}

Outcome data are summarized in Table 4. Clinical cure was achieved in $43.0 \%$ of patients in an average time of approximately three weeks. Severe sepsis or septic shock was present in $28.0 \%$ of patients during index hospitalization. Of the 61 patients who survived their index hospitalization, 
$26.3 \%(16 / 61)$ were readmitted within 60 days, with a mean time from discharge to readmission of 56.9 days $(\mathrm{SD}=51.9)$. Of these patients, recurrence of infection was the cause of readmission in $6.25 \%$ of patients. Half of patients readmitted had "other" (including cardiovascular, gastrointestinal or metabolic reason) or unknown reason recorded in their notes. Mortality was observed in

Supplementary Table $\mathbf{5 a}$ - Antibacterial treatments received during the study period.

\begin{tabular}{|c|c|c|c|}
\hline & $\begin{array}{l}\text { Immediately after } \\
\text { culture collection* }\end{array}$ & $\begin{array}{l}\text { Immediately prior to receipt } \\
\text { of susceptibility results }{ }^{* *}\end{array}$ & $\begin{array}{c}\text { After receipt of } \\
\text { susceptibility results }\end{array}$ \\
\hline & $(N=100)$ & $(N=100)$ & $(N=100)$ \\
\hline Aminoglycosides - amikacin & $0(0.0 \%)$ & $0(0.0 \%)$ & $1(1.0 \%)$ \\
\hline Aminoglycosides - gentamicin & $1(1.0 \%)$ & $8(8.0 \%)$ & $14(14.0 \%)$ \\
\hline Aminoglycosides - tobramycin & $1(1.0 \%)$ & $1(1.0 \%)$ & $1(1.0 \%)$ \\
\hline Carbapenems - ertapenem & $0(0.0 \%)$ & $1(1.0 \%)$ & $4(4.0 \%)$ \\
\hline Carbapenems - imipenem/cilastatin & $1(1.0 \%)$ & $2(2.0 \%)$ & $3(3.0 \%)$ \\
\hline Carbapenems - meropenem & $18(18.0 \%)$ & $41(41.0 \%)$ & $61(61.0 \%)$ \\
\hline Cephalosporins - cefepime & $1(1.0 \%)$ & $1(1.0 \%)$ & $0(0.0 \%)$ \\
\hline Cephalosporins - ceftazidime & $0(0.0 \%)$ & $1(1.0 \%)$ & $1(1.0 \%)$ \\
\hline Cephalosporins - ceftolozane/tazobactam & $7(7.0 \%)$ & $7(7.0 \%)$ & $2(2.0 \%)$ \\
\hline Cephalosporins - ceftriaxone & $0(0.0 \%)$ & $1(1.0 \%)$ & $0(0.0 \%)$ \\
\hline Cephalosporins - cefuroxime & $1(1.0 \%)$ & $0(0.0 \%)$ & $0(0.0 \%)$ \\
\hline Colistin & $14(14.0 \%)$ & $25(25.0 \%)$ & $61(61.0 \%)$ \\
\hline Daptomycin & $2(2.0 \%)$ & $2(2.0 \%)$ & $2(2.0 \%)$ \\
\hline Fluoroquinolones - ciprofloxacin & $2(2.0 \%)$ & $3(3.0 \%)$ & $2(2.0 \%)$ \\
\hline Fluoroquinolones - levofloxacin & $6(6.0 \%)$ & $8(8.0 \%)$ & $2(2.0 \%)$ \\
\hline Fluoroquinolones - lomefloxacin & $1(1.0 \%)$ & $1(1.0 \%)$ & $0(0.0 \%)$ \\
\hline Fluoroquinolones - moxifloxacin & $1(1.0 \%)$ & $1(1.0 \%)$ & $0(0.0 \%)$ \\
\hline Fosfomycin & $0(0.0 \%)$ & $3(3.0 \%)$ & $8(8.0 \%)$ \\
\hline Glycopeptides - vancomycin & $2(2.0 \%)$ & $4(4.0 \%)$ & $2(2.0 \%)$ \\
\hline Glycopeptides- teicoplanin & $5(5.0 \%)$ & $7(7.0 \%)$ & $4(4.0 \%)$ \\
\hline Glycylcycline - tigecycline & $2(2.0 \%)$ & $8(8.0 \%)$ & $13(13.0 \%)$ \\
\hline Linezolid & $6(6.0 \%)$ & $6(6.0 \%)$ & $4(4.0 \%)$ \\
\hline Metronidazole & $1(1.0 \%)$ & $1(1.0 \%)$ & $1(1.0 \%)$ \\
\hline Nitrofurantoin & $0(0.0 \%)$ & $0(0.0 \%)$ & $1(1.0 \%)$ \\
\hline Penicillins - amoxicillin & $0(0.0 \%)$ & $0(0.0 \%)$ & $1(1.0 \%)$ \\
\hline Penicillins - ampicillin & $0(0.0 \%)$ & $1(1.0 \%)$ & $0(0.0 \%)$ \\
\hline Penicillins - piperacillin & $14(14.0 \%)$ & $16(16.0 \%)$ & $5(5.0 \%)$ \\
\hline Tetracyclines - minocycline & $1(1.0 \%)$ & $1(1.0 \%)$ & $1(1.0 \%)$ \\
\hline Tetracyclines - tetracycline & $0(0.0 \%)$ & $1(1.0 \%)$ & $0(0.0 \%)$ \\
\hline Trimethoprim - sulfamethoxazole & $0(0.0 \%)$ & $1(1.0 \%)$ & $3(3.0 \%)$ \\
\hline
\end{tabular}

*First antibacterial treatment(s) the patient received the next day after culture collection. If date of culture collection was the same as the date of susceptibility test result availability, the following definition was applied: treatment for which start date was before date of susceptibility result availability and date of finalization was after or up to two days prior to the date of susceptibility result availability.

**Treatment for which start date was before date of susceptibility result availability and date of finalization was after or up to two days prior to the date of susceptibility result availability.

***Treatments for which start date was after the date of susceptibility result availability and no later than two days after the date of susceptibility result availability. Treatment start date was prior to the date of susceptibility result availability and treatment end date was at minimum two days after the date of susceptibility result availability. 


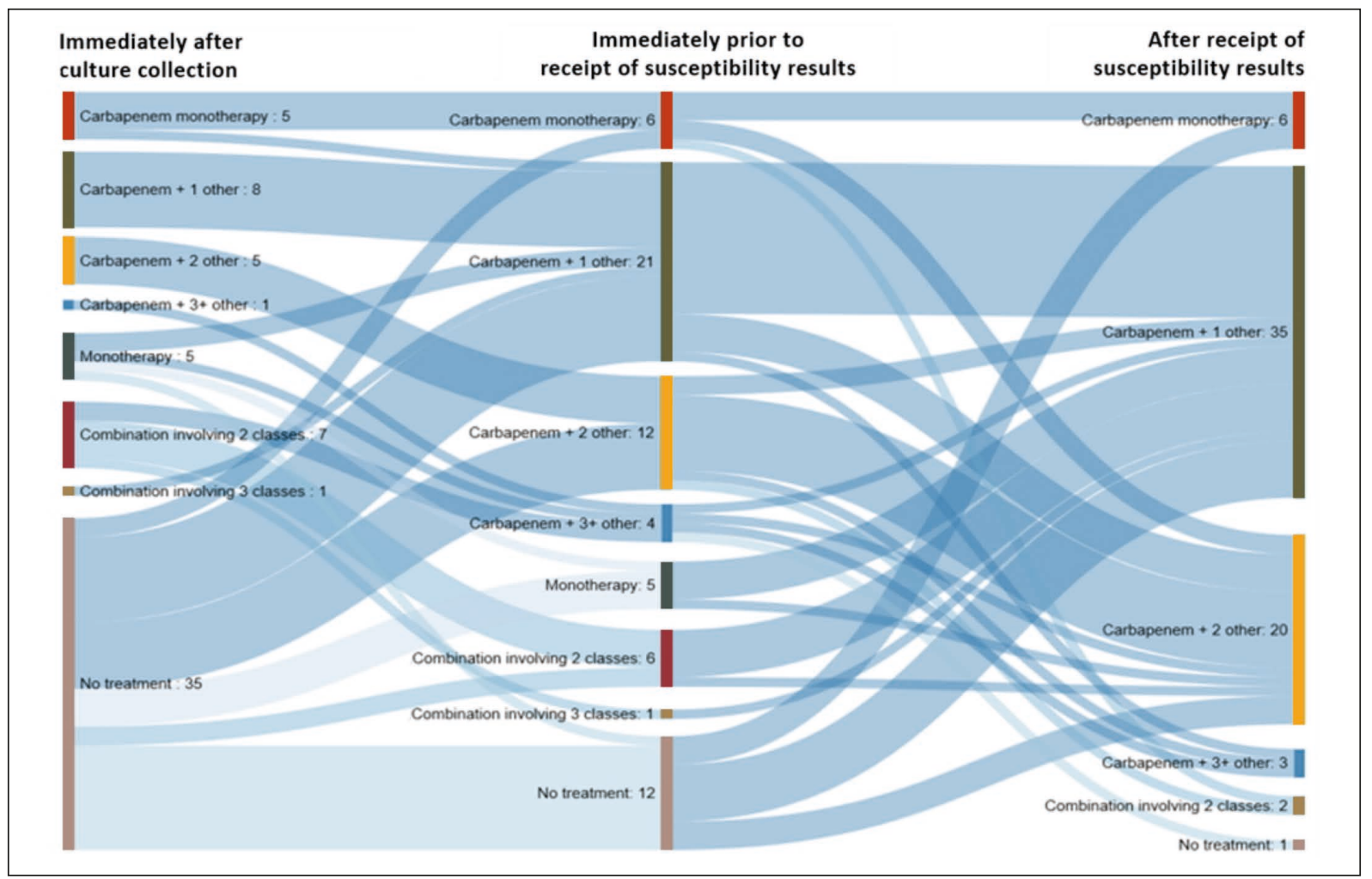

Figure 5 - Sankey figure of treatment pathways for patients who received a carbapenem during observation period.

Supplementary Table $5 \mathbf{b}$ - Antibacterial regimens received during the study period for patients who received carbapenem at any time during the study period.

\begin{tabular}{|c|c|c|c|}
\hline & $\begin{array}{l}\text { Immediately after culture } \\
\text { collection }\end{array}$ & $\begin{array}{l}\text { Immediately prior to receipt } \\
\text { of susceptibility results*** }\end{array}$ & $\begin{array}{l}\text { After receipt of susceptibility } \\
\text { results }\end{array}$ \\
\hline & $(N=67)$ & $(N=67)$ & $(N=67)$ \\
\hline Carbapenem monotherapy & $5(7.5 \%)$ & $6(9.0 \%)$ & $6(9.0 \%)$ \\
\hline Carbapenem +1 other & $8(11.9 \%)$ & $21(31.3 \%)$ & $35(52.2 \%)$ \\
\hline Carbapenem +2 other & $5(5.3 \%)$ & $12(17.9 \%)$ & $20(29.9 \%)$ \\
\hline Carbapenem $+3+$ other & $1(1.5 \%)$ & $4(6.0 \%)$ & $3(4.5 \%)$ \\
\hline Monotherapy & $5(7.5 \%)$ & $5(7.5 \%)$ & $0(0 \%)$ \\
\hline Combination involving 2 classes & $7(10.5 \%)$ & $6(9.0 \%)$ & $2(3.0 \%)$ \\
\hline Combination involving 3 classes & $1(1.6 \%)$ & $1(1.6 \%)$ & $0(0.0 \%)$ \\
\hline Combination involving $4+$ classes & $0(0.0 \%)$ & $0(0.0 \%)$ & $0(0.0 \%)$ \\
\hline No treatment & $35(52.2 \%)$ & $12(17.9 \%)$ & $1(1.6 \%)$ \\
\hline
\end{tabular}

${ }^{*}$ First antibacterial treatment(s) the patient received the next day after culture collection. If date of culture collection was the same as the date of susceptibility result availability, the following definition was applied: treatment for which start date was before date of susceptibility result availability and date of finalization was after or up to two days prior to the date of susceptibility result availability.

**Treatment for which start date was before date of susceptibility result availability and date of finalization was after or up to two days prior to the date of susceptibility result availability.

***Treatments for which start date was after the date of susceptibility result availability and no later than two days after the date of susceptibility result availability. Treatment start date was prior to the date of susceptibility result availability and treatment end date was at minimum two days after the date of susceptibility result availability. 
Table 3 - Healthcare resource use: percentage of patients who underwent diagnostic tests and/or procedures during index hospitalization.

\begin{tabular}{|l|c|}
\hline \multicolumn{1}{|c|}{ Test or procedure } & No. $(\%)$ \\
\hline Diagnostic test and/or procedure during & \\
index hospitalization & \\
Blood cell count & $99(99.0 \%)$ \\
Biochemistry test & $78(78.0 \%)$ \\
Blood culture & $67(67.0 \%)$ \\
Radiograph & $46(46.0 \%)$ \\
Urine culture & $44(44.0 \%)$ \\
Bronchoscopy & $36(36.0 \%)$ \\
CT & $29(29.0 \%)$ \\
ECG & $22(22.0 \%)$ \\
Sputum analysis & $16(16.0 \%)$ \\
Other diagnostic test & $3(3.0 \%)$ \\
\hline Invasive procedure during index & \\
hospitalization & \\
Endoscopy & $19(19.0 \%)$ \\
Vascular catheter & $15(15.0 \%)$ \\
Percutaneous drainage & $9(9.0 \%)$ \\
Surgery (incl. surgical drainage) & $5(5.0 \%)$ \\
Urinary catheter & $3(3.0 \%)$ \\
Mechanical ventilation & $2(2.0 \%)$ \\
Cardiac catheterization & $2(2.0 \%)$ \\
\hline
\end{tabular}

Note: Table shows diagnostic tests and invasive procedures performed during index hospitalization and diagnostic tests in the follow up period for patients who have reported at minimum one diagnostic test related to infection treatment from 6 months after discharge (data regarding invasive procedures in this period was not available). CT: computed tomography, ECG: electrocardiogram.
$39.0 \%$ of included patients during their index hospitalization, with a mortality rate of $65.0 \%$ by the end of the follow-up period. The most common cause of death was deemed to be infection related.

\section{DISCUSSION}

The aim of this study was to assess the clinical management of patients diagnosed with cUTI, cIAI, and HABP/VABP resulting from CRGN bacteria in Italian hospitals. In the hospital survey we found that CR occurred in about $17 \%$ of the complicated infections in focus and that A. baumannii had the highest rates of CR. Additionally, in the retrospective study we found that complicated CRGN infections were hospital- or healthcare-acquired in $99 \%$ of cases and were most often caused by K. pneumoniae. Despite some use of rapid diagnostic procedures and frequent utilization of antibiotic combination treatments, outcomes were relatively poor - with an average length of hospital stay of over 50 days, achievement of clinical cure in less than half of patients, and an overall mortality rate of $65.0 \%$. Due to the low number of patients $(n=14)$ it was not possible to analyze mortality according to ICU and medical/surgical wards. These outcomes are in line with previously

Table 4 - Outcomes.

\begin{tabular}{|l|c|}
\hline \multicolumn{1}{|c|}{ Characteristic } & No. (\%) \\
\hline Clinical cure achieved* & $43(43.0 \%)$ \\
Time to clinical cure from culture collection - days - mean (SD) & $20.6(11.6)$ \\
Severe sepsis/septic shock & $28(28.0 \%)$ \\
Duration of severe sepsis/septic shock - days - mean (SD) & $4.5(3.8)$ \\
Mortality during index hospitalization & $39(39.0 \%)$ \\
Time to discharge from culture collection - days - mean (SD) & $33.8(52.5)$ \\
Time to ICU discharge since culture collection - days - mean (SD) & $8.3(14.6)$ \\
Time to death since culture collection - days - mean (SD) & $134.9(205.2)$ \\
Time to readmission from discharge following index hospitalization - days - mean (SD) $)^{* * *}$ & $56.9(51.9)$ \\
Readmission rate at 30 days** & $5(8.8 \%)$ \\
Readmission rate at 60 days* & $16(26.3 \%)$ \\
Overall mortality (at chart review) & $65(65.0 \%)$ \\
\hline Cause of death & \\
Infection-related & $28(43.1 \%)$ \\
Other & $16(24.6 \%)$ \\
Unknown & $21(32.3 \%)$ \\
\hline
\end{tabular}

*As defined by complete resolution of all signs and symptoms of infection.

** Readmission rate was calculated using the number of alive patients at discharge as denominator.

***Time to readmission since discharge was calculated for all patients who reported a hospitalization up to 6 months after hospital discharge. Those patients with missing information about status (alive or deceased) at 6 months following hospital discharge were removed from the population used to assess readmission, in order to avoid a potential bias to higher hospitalization rates. 
published international data on CRGN infections, such as patients with K. pneumoniae infections and patients with CR Pseudomonas bacteraemia [1, 11]. Appropriate (both effective and timely) antimicrobial therapy is known to improve the outcome of infections while being consistent with the principles of antimicrobial stewardship [12, 13]. Important attributes of appropriate treatment are the selection of effective empirical therapy, identification (ideally rapid) of the causative bacteria and antibiotic susceptibility, and subsequent timely modifications to prescribed treatments to ensure appropriate definitive therapy is being given. CRGN infections pose a particularly difficult challenge for healthcare providers trying to comply with antimicrobial stewardship principles, due to the higher chance of selecting an initial therapy which is inappropriate and the associated delay to appropriate treatment [13].

Considering the need to identify bacteria and antibiotic susceptibility properly, our study showed that bacterial identification in Italian hospitals was often achieved through MALDI-TOF, a rapid procedure previously reported to significantly reduce time to optimization of antibiotic therapy as well as reducing hospital costs [14]. Additionally, susceptibility verification was predominantly performed by automated susceptibility testing, a technique also associated with shorter diagnostic times [15]. On the other hand, according to the results of our cohort, PCR-based identification methods were not used extensively, and carbapenemase testing was limited to endemic KPC identification. Since the identification of carbapenemase type can help to optimize therapy, increased availability of such diagnostic methods might lead to improved prescribing choices and infection outcomes.

In this study we observed that 19 patients received a carbapenem as an early empirical therapy, 43 as a late empirical therapy, and 64 as part of the treatment regimen even after receiving the susceptibility data confirming that the infection was due to CRGN bacteria. This observation may explain some of the poor outcomes reported in our study cohort and indicates a need for additional therapeutic options for the management of CRGN infections in the Italian setting. Treatment pattern analysis performed in our study for patients receiving carbapenems at any timepoint describes trends in early and late empirical and targeted an- tibacterial therapy for these difficult-to-treat infections. Initial carbapenem therapy was observed in $19.0 \%$ of this subset of patients either as a monotherapy or in combination, which is in line with the $16.6 \%$ figure previously reported for CR Enterobacterales bloodstream infections in Italy [16]. However, overall carbapenem use was substantial, since they were prescribed - predominantly in combination with other antibiotics - in $43.0 \%$ of patients as late empirical therapy and in $64.0 \%$ of patients as targeted therapy. When looking at all patients, the most frequent concomitant therapy was colistin, perhaps due to the need for a broad coverage agent that can address the prevalent KPC-producers in Italy (recent evidence suggests that KPC-producing K. pneumoniae infections can be treated adequately with colistin-carbapenem combinations [17]. Colistin is associated with various negative consequences, including renal toxicity and suboptimal dosing, but may be utilized for these complicated infections due to the historical dearth of alternative effective agents [18]. However, even combination regimens, including colistin-based combinations, may not lead to better outcomes $[19,20]$. This is reflected in our cohort, where clinical cure was relatively low and overall mortality high, despite use of combination therapy and last resort agents.

Other possible explanations for the poor outcomes observed in the study cohort relate to known patient-associated risk factors for worse outcomes, namely high levels of previous exposure to healthcare environments where antibiotic-resistant bacteria are likely to be present and the selection pressures which may be exerted by previous antibiotic administration [21]. Indeed, nearly all $(99.0 \%)$ CRGN complicated infections were found to be healthcare-associated in our cohort, 57 patients had a documented hospital admission in the previous year and 38 had received prior antibiotic treatment, including 11 who were prescribed a carbapenem treatment. The identification of these risk factors within this Italian cohort supports the importance of considering these factors when evaluating the possibility of CRGN infections and planning empirical therapy, bypassing carbapenems in favor of agents which will address CR.

The strengths of the EU-CARE study include an extensive analysis of the management in Italy of complicated CRGN infections as well as new in- 
formation on the real-world treatment patterns of patients with these difficult to treat conditions. Our study, however, has certain limitations as well. The small number of sites prohibits the generalization of results to the whole of the country, although survey data from the study sites is aligned with epidemiological data previously published for Italy [3]. The retrospective chart review data collection method assumed equivalent definitions regarding clinical terms and microbiological methods (e.g. dilution, disk diffusion, automated antimicrobial susceptibility test). However, these definitions were neither confirmed nor harmonized, which might have contributed to an unmeasured variance in the results. Additionally, reasons for clinical decision-making in the administration of antibiotics were not assessed in our study, although these might be important aspects of optimizing antimicrobial stewardship while treating infections caused by resistant bacteria. Furthermore, the chart review method might have resulted in missing data, which might explain some unexpected findings in the healthcare resource variables, i.e. that the rates of invasive procedures during index hospitalizations were reportedly low and that mechanical ventilation was only reported in $2.0 \%$ of cases despite $56.0 \%$ of the cases being HABP/VABP and $44.0 \%$ of patients requiring time in the ICU).

The literature also reports changes in resistance mechanisms among Enterobacteriaceae within some Italian hospitals over time [22]. Therefore, the findings of this study should be considered in context of their institutional setting and time frame of the data reported. Furthermore, in response to changing epidemiology and the availability of novel agents, it is important to recognize changes in clinical management that have not been captured within this study. In recent years new non-colistin-based regimens (including ceftolozane-tazobactam, ceftazidime-avibactam and/or meropenem-vaborbactam) have also been introduced into clinical practice impacting clinical outcomes and also local ecologies [23]. Between 2015 and 2019, there were significantly increasing trends in the European population-weighted mean percentages for carbapenem resistance. Across Europe, Italy was one of the regions with largest percentage of invasive $K$. pneumoniae isolates resistant to carbapenems [24]. This resistance situation remains problematic. The time period used for the study and the changes seen during the last years may affect the generalizability of our results to the current epidemiology and clinical management of these infections in Italy. In terms of type of infections, the epidemiological profile of each region is of major importance in order to define the most appropriate treatment algorithm. A recently published cross-sectional survey showed that the treatment of CRGN infections is highly variable with clinicians using a wide range of antibacterial strategies and combinations informed by clinical severity, local availability, and clinical experience [25]. Given the availability of novel non-colistin-based treatment regimens it is important to recognize their optimal use within the treatment pathway, and when possible inform treatment choice based on pathogen susceptibility profiles [23].

Finally, due to recent changes in the EUCAST definitions (i.e. for testing categories S, I, and R), our study might have included cases that would not currently qualify as CR [26].

In conclusion, our results reflect the multi-faceted medical burden associated with complicated CRGN infections. Our study pinpoints potential areas for improvement in treatment practices. For example, regular and detailed local surveillance and use of accurate and rapid microbial diagnostic techniques might aid clinical decision-making and facilitate improvements in antimicrobial stewardship when treating CRGN infections. Further research is needed to identify new therapeutic options, and to use the novel agents which are targeted to the treatment of CRGN infections, in order to optimize patient treatment and improve overall patient outcomes.

\section{Declaration of interest and funding}

This study was funded by Merck Sharp \& Dohme Corp. (MSD), a subsidiary of Merck \& Co., Inc., Kenilworth, NJ, USA. Eilish McCann was full time employee of Merck \& Co., Inc., Kenilworth, NJ, USA at the time of the study conduct and reports personal fees from Merck \& Co., Inc., outside the submitted work. Rita Citton is full time employees of MSD, Rome, Italy. IQVIA, employer of David Gómez-Ulloa and Montse Roset, received consulting fees from Merck \& Co., Inc. Dr. Durante-Mangoni reports grants from MSD and Pfizer, personal fees from Pfizer, MSD, Angelini, 
Nordic Pharma, Sanofi-Aventis, Roche and Correvio, outside of the submitted work. Dr. Mastroianni reports grants from MSD. Dr. Bassetti reports personal fees and other from Angelini, Astellas, Bayer, bioMérieux, Cidara, Gilead, Menarini, MSD, Nabriva, Pfizer and Shionogi, outside the submitted work. Dr. Bertolino and Dr. Viale reported no conflict of interest to declare.

\section{Acknowledgements}

The authors would like to thank the medical communications agency EpiConsult for their assistance in the preparation of the manuscript, tables, and figures and Dr. Alessandra Oliva for her contribution in the acquisition of the study data.

\section{REFERENCES}

[1] Agyeman AA, Bergen PJ, Rao GG, Nation RL, Landersdorfer CB. A systematic review and meta-analysis of treatment outcomes following antibiotic therapy among patients with carbapenem-resistant Klebsiella pneumoniae infections. Int J Antimicrob Agents. 2020; 55 (1), 105833.

[2] Rogers Van Katwyk S, Grimshaw JM, Nkangu M, Nagi R, Mendelson M, Taljaard M, Hoffman SJ. Government policy interventions to reduce human antimicrobial use: A systematic review and evidence map. PLoS Medicine. 2019; 16 (6), e1002819.

[3] Surveillance of antimicrobial resistance in Europe [https://www.ecdc.europa.eu/en/publications-data / surveillance-antimicrobial-resistance-europe-2017].

[4] Global priority list of antibiotic-resistant bacteria to guide research, discovery, and development of new antibiotics [https://www.who.int/medicines/publications/WHO-PPL-Short_Summary_25Feb-ET_NM_ WHO.pdf?ua=1].

[5] Giani T, Pini B, Arena F, et al. Epidemic diffusion of KPC carbapenemase-producing Klebsiella pneumoniae in Italy: results of the first countrywide survey, 15 May to 30 June 2011. Euro Surveill. 2013; 18 (22).

[6] Iacchini S, Sabbatucci M, Gagliotti C, et al. Bloodstream infections due to carbapenemase-producing Enterobacteriaceae in Italy: results from nationwide surveillance, 2014 to 2017. Euro Surveill. 2019; 24 (5).

[7] Monaco M, Giani T, Raffone M, et al. Colistin resistance superimposed to endemic carbapenem-resistant Klebsiella pneumoniae: a rapidly evolving problem in Italy, November 2013 to April 2014. Euro Survell. 2014; 19 (42).

[8] Problems with colistin susceptibility testing and several commercially available products [https://eucast.org/ast_of_bacteria/warnings/].

[9] Marquet K, Liesenborgs A, Bergs J, Vleugels A, Claes
$\mathrm{N}$. Incidence and outcome of inappropriate in-hospital empiric antibiotics for severe infection: a systematic review and meta-analysis. Crit care (London, England). 2015; 19 (1), 63.

[10] Breakpoint tables for interpretation of MICs and zone diameters. [https://www.eucast.org/fileadmin/ $\mathrm{src} /$ media/PDFs/EUCAST_files/Breakpoint_tables/v_7.1_Breakpoint_Tables.pdf].

[11] Zhang Y, Chen XL, Huang AW, et al. Mortality attributable to carbapenem-resistant Pseudomonas aeruginosa bacteremia: a meta-analysis of cohort studies. Emerg Micr Infect. 2016; 5 (3), e27.

[12] Rhodes A, Evans LE, Alhazzani W, et al. Surviving sepsis campaign: international guidelines for management of sepsis and septic shock: 2016. Intensive Care Med. 2017; 43 (3), 304-77.

[13] Johnston ANB, Park J, Doi SA, Sharman V, Clark J, Robinson J, Crilly J. Effect of immediate administration of antibiotics in patients with sepsis in tertiary care: a systematic review and meta-analysis. Clin Ther. 2017; 39 (1), 190-202.e196.

[14] Perez KK, Olsen RJ, Musick WL, et al. Integrating rapid pathogen identification and antimicrobial stewardship significantly decreases hospital costs. Arch Path Lab Med. 2013; 137 (9), 1247-54.

[15] Mittman SA, Huard RC, Della-Latta P, Whittier S. Comparison of BD phoenix to vitek 2, microscan MICroSTREP, and Etest for antimicrobial susceptibility testing of Streptococcus pneumoniae. J Clin Microbiol. 2009; 47 (11), 3557-61.

[16] Harris PNA, Pezzani MD, Gutiérrez-Gutiérrez B, et al. Geographical variation in therapy for bloodstream infections due to multidrug-resistant Enterobacteriaceae: a post-hoc analysis of the INCREMENT study. Int $J$ Antimicrob Agents. 2017; 50 (5), 664-72.

[17] Hawkey PM, Warren RE, Livermore DM, et al. Treatment of infections caused by multidrug-resistant Gram-negative bacteria: report of the British Society for Antimicrobial Chemotherapy/Healthcare Infection Society/British Infection Association Joint Working Party. J Antimicron Chemother. 2018; 73 (Suppl_3), iii2-iii78.

[18] Lim LM, Ly N, Anderson D, et al. Resurgence of colistin: a review of resistance, toxicity, pharmacodynamics, and dosing. Pharmacother. 2010; 30 (12), 1279-91. [19] Paul M, Daikos GL, Durante-Mangoni E, et al. Colistin alone versus colistin plus meropenem for treatment of severe infections caused by carbapenem-resistant Gram-negative bacteria: an open-label, randomised controlled trial. The Lancet Infect Dis. 2018; 18 (4), 391-400.

[20] Shi H, Lee JS, Park SY, Ko Y, Eom JS. Colistin Plus Carbapenem versus Colistin Monotherapy in the Treatment of Carbapenem-Resistant Acinetobacter baumannii Pneumonia. Infect Drug Res. 2019; 12, 3925-34.

[21] Timsit JF, Bassetti M, Cremer O, et al. Rationalizing antimicrobial therapy in the ICU: a narrative review. Intensive Care Med. 2019, 45 (2), 172-89. 
[22] Tavoschi L, Forni S, Porretta A, et al. on behalf of the Tuscan Clinical Microbiology Laboratory Network. Prolonged outbreak of New Delhi metallo-beta-lactamase-producing carbapenem-resistant Enterobacterales (NDM-CRE), Tuscany, Italy, 2018 to 2019. Euro Surveill. 2020;25(6):pii=2000085. https://doi. org/10.2807/1560-7917.ES.2020.25.6.2000085

[23] Puzniak L, Dillon R, Palmer T, Collings H, Enstone A. Real-world use of ceftolozane/tazobactam: a systematic literature review. Antimicrob Resist Infect Control. 2021; 8; 10 (1), 68.

[24] European Centre for Disease Prevention and Con- trol. Antimicrobial resistance in the EU/EEA (EARSNet) - Annual Epidemiological Report 2019. Stockholm: ECDC; 2020.

[25] Carrara E, Savoldi A, Piddock LJV, et al. Clinical management of severe infections caused by carbapenem-resistant gram-negative bacteria: a worldwide cross-sectional survey addressing the use of antibiotic combinations. Clin Microbiol Infect. 2021; 8, S1198$743 \times(21) 00222-6$.

[26] The European Committee on Antimicrobial Susceptibility Testing. S, I and R definitions. [https:// www.eucast.org/newsiandr/]. 